\title{
The size matters: regulation of lipid storage by lipid droplet dynamics
}

\author{
Jinhai Yu \& Peng $\mathrm{Li}^{*}$ \\ Tsinghua-Peking Center for Life Sciences, School of Life Sciences, Tsinghua University, Beijing 100084, China
}

Received October 23, 2016; accepted October 28,2016; published online December 5, 2016

\begin{abstract}
Adequate energy storage is essential for sustaining healthy life. Lipid droplet (LD) is the subcellular organelle that stores energy in the form of neutral lipids and releases fatty acids under energy deficient conditions. Energy storage capacity of LDs is primarily dependent on the sizes of LDs. Enlargement and growth of LDs is controlled by two molecular pathways: neutral lipid synthesis and atypical LD fusion. Shrinkage of LDs is mediated by the degradation of neutral lipids under energy demanding conditions and is controlled by neutral cytosolic lipases and lysosomal acidic lipases. In this review, we summarize recent progress regarding the regulatory pathways and molecular mechanisms that control the sizes and the energy storage capacity of LDs.
\end{abstract}

lipid storage, lipid droplet, TAG synthesis, atypical LD fusion, lipolysis

Citation: Yu, J., and Li, P. (2017). The size matters: regulation of lipid storage by lipid droplet dynamics. Sci China Life Sci 60, 46-56. doi: 10.1007/s11427-016$0322-\mathrm{x}$

\section{INTRODUCTION}

Energy is essential for life as it can be converted to ATP to perform meaningful work at an acceptable metabolic cost in healthy organism. Food, the major chemical source of energy, is digested and absorbed through the digestive system. Part of the energy is stored in cells and can be transformed and utilized to sustain life under starvation, prolonged physical activity and stress conditions. Glycogen and triacylglycerol (TAGs, neutral lipids or fat) are the two major forms of stored energy in the body. Glycogen is mainly stored in the liver and muscle. TAG, the most efficient form of energy due to its high energy density and the hydrophobic characteristics, is mainly stored in the adipose tissue, whereas small amounts are found in the liver and muscle (Herman, 2016). The amount of stored energy represents the balance between energy intake and energy expenditure.

*Corresponding author (email: 1i-peng@tsinghua.edu.cn)
The subcellular organelle responsible for lipid storage is lipid droplet (LD) that is present in most organisms and cell types (Murphy, 2012). Adipose tissue is the main tissue responsible for lipid storage in higher animals. Mammals mainly possess two types of adipose tissue: white and brown adipose tissue (WAT and BAT, respectively). White adipocyte contains a unilocular LD and is the major lipid storage cell in the body. LD stores excess energy in the form of TAG and releases free fatty acids (FFAs) by TAG degradation. Besides being actively involved in lipid storage and lipid homeostasis, LDs are highly dynamic in the cell by interacting with many other subcellular organelles including endoplasmic reticulum (ER), mitochondria, lysosome, endosome and autophagosome (Barbosa et al., 2015; Beller et al., 2010; Fujimoto and Parton, 2011; Murphy et al., 2009; Welte, 2015; Zehmer et al., 2009). The lipid storage capacity of LDs is determined by its "size" (the volume) and controlled by the balance beween TAG synthesis and degradation, and also CIDE (cell death-inducing DFFA (DNA fragmentation 
factor, alpha subunit)-like effector) family proteins mediated atypical LD fusion. Net increase of cellular or localized TAG synthesis or CIDE mediated atypical LD fusion promotes LD growth and expansion. On the contrary, TAG degradation via cytosol or lysosomal lipase results in the reduction of LD sizes and lower lipid storage capacity. Regulation of LD sizes and lipid storage capacity is important in maintaining normal biological function especially under starvation and stress conditions. Its deregulation results in the development of metabolic diseases including obesity, diabetes, fatty liver, cardiovascular disease, neurodegenerative disease and cancer (Filipe and McLauchlan, 2015; Gong et al., 2009; Gross and Silver, 2014; Krahmer et al., 2013; Liu et al., 2015; Rutkowski et al., 2015; Saka and Valdivia, 2012; Suzuki et al., 2011; Xu et al., 2012). This review summarizes the structure and function of LDs and the main regulatory pathways and molecular mechanism that control the sizes of LDs and the lipid storage capacity.

\section{THE STRUCTURE AND FUNCTION OF LDS}

LD is composed of a monolayer of phospholipids, a neutral lipid core and various associated proteins (Figure 1). Lipidomic analyses of isolated LDs from various cell types reveal that phosphatidylcholine (PC) is the major phospholipid, followed by phosphatidylethanolamine (PE) and phosphatidylinositol (PI). Small amount of lyso-PC and lyso-PE, ether-linked form of $\mathrm{PC}$ and $\mathrm{PE}$ and free cholesterol has also been detected in LD fraction (Bartz et al., 2007;
Ohsaki et al., 2014; Tauchi-Sato et al., 2002; Walther and Farese, 2012). Plin1 is the first LD-associated protein identified in mammalian cells. Since then, many types of LD associated proteins are identified by various proteomic and molecular analyses. Majority of the LD-associated proteins are involved in controlling LD dynamics under different nutrient conditions and hormone stimulations. The main components of neutral lipid core in most eukaryotic cells are TAG and sterol ester (SE). Ribosome, ER-like membrane and other proteins are also reported in the neutral lipid core in specific cell types (Robenek et al., 2009; Wan et al., 2007). The actual structure or organization of the neutral lipid core remains to be elusive. FFAs released from LDs by TAG degradation can function as potential ligands for PPARs and substrates for synthesis of other signal molecules, such as endocannabinoids and prostaglandins (Zechner et al., 2012). Neutral lipids stored in LDs also detoxify FFAs and cholesterols, and as a key reservoir of membrane components and energy, which confers selective advantages to the organisms.

In eukaryotes, LDs are generated from ER where most of enzymes for TAG synthesis are localized. Due to the physical feature, nascent LDs are budded out from ER membrane and form cytosolic LDs. However, cytosol LDs are observed to have extensive contacts with ER and LD-ER contacts may act as a conduit for the lipids and proteins exchange, and may related to ER stress and ER-assisted degradation (ERAD) of unfolded proteins (Barbosa et al., 2015; Gao and Goodman, 2015; Jacquier et al., 2011; Kassan et al., 2013; Knoblach and Rachubinski, 2015; Wilfling et al., 2014). Mitochondria and

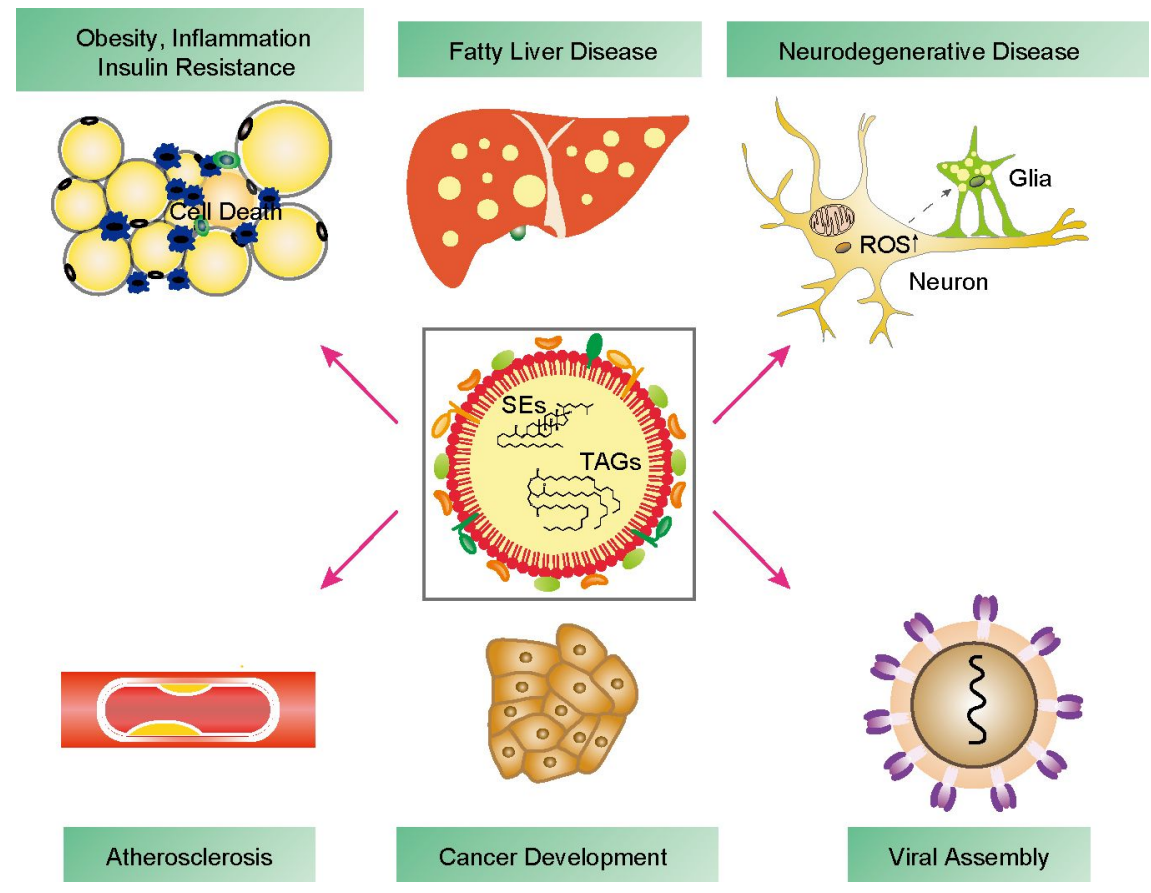

Figure 1 The structure and function of LDs. LD is composed of a neutral lipid core, a monolayer phospholipid membrane and its associated surface proteins. The major components of neutral lipids in mammalian LD are triacylglycerols (TAGs) and sterol esters (SEs). Dysregulated LD homeostasis is closely connected to many pathological conditions and the development of various diseases reactive oxygen species (ROS). 
peroxisome, organelles responsible for lipid oxidation, also have close association with LDs, and Plin5 has been identified to mediate the interaction between LD and mitochondria and may facilitate the transport of FA for oxidative phosphorylation with increased energy demand (Gao and Goodman, 2015; Lodhi and Semenkovich, 2014; Mason and Watt, 2015; Pu et al., 2011; Rambold et al., 2015; Schrader, 2001; Yu et al., 2015). LDs are also observed surrounding the nucleus or actually present in the nucleus that may function to store specific proteins or sequester transcriptional factors (Farese and Walther, 2016; Welte, 2015). The interaction between LDs and endosomes also observed and likely regulated by Rab5 (Liu et al., 2007).

Excess or insufficient lipid storage is associated with many pathological conditions including obesity, insulin resistance, inflammation, fatty liver diseases, atherosclerosis, neurodegenerative disease, cancer development and viral replication (Figure 1). As the major tissue for lipid storage, adipose tissue plays a crucial role in controlling lipid homeostasis. It protects the body from lipotoxity by transforming FFAs into TAGs, and releases glycerol and FFAs as energy resource for other tissues during energy limitation. Excessive lipid storage in adipocytes results in hypoxia, ER stress, insufficient angiogenesis, increased adipocyte death, high immune cell infiltration, increased secretion of pro-inflammatory cytokines and the development of obesity and insulin resistance (Sell et al., 2012; Ye, 2013). On the other hand, insufficient lipid storage in adipose tissue under lipodystrophic condition leads to adipocyte lipotoxicity, mitochondrial dysfunction, increased oxidative stress, inflammatory responses, deregulated release of free fatty acids and impaired insulin sensitivity (Bindlish et al., 2015; Frayn, 2001; Grundy, 2015).

Excess lipid storage in the form of cholesterol esters in LDs of macrophages due to the increased uptake of oxidized lowdensity lipoprotein is the major cause of foam cell formation during the development of atherosclerosis and cardiovascular disease. Higher lipid storage and increased LD sizes in leukocytes is observed under allergic inflammation and pathogen infections. Degradation of TAG from LDs in leukocytes by lipases (Dichlberger et al., 2014; Melo and Weller, 2016) liberates arachidonic acid (AA) that is further converted to prostaglandin $(\mathrm{PG})$ by cyclooxygenase $(\mathrm{COX})$ in response to immuno-activation. LDs are also shown to be associated with viral assembly of hepatitis $\mathrm{C}$ virus (HCV), dengue virus, bacterial infection, and anti-pathogen infection due to its association with Histone proteins and Viperin (Anand et al., 2012; Camus et al., 2013; Crawford and Desselberger, 2016; D’Avila et al., 2008; Filipe and McLauchlan, 2015; Hinson and Cresswell, 2009). Most recent research has demonstrated that the size of LDs and increased lipid accumulation is associated with cancer development and neural degenerative diseases (Baenke et al., 2013; Chen and Li, 2016; Liu et al., 2015; Qiu et al., 2015).

\section{INCREASED LIPID STORAGE BY THE GROWTH OF LDS}

The sizes of LDs vary drastically in different cell types, reflecting their varying lipid storage capacity. The diameters of LDs in most non-adipose cells range from dozens of nanometers to several micrometers. In white adipocytes that are most efficient in storing lipids, the diameters of their uniloculars LD can be easily larger than $100 \mu \mathrm{m}$. As unilocular large LD contains smaller surface area compared with that of multilocular smaller LDs, it is protected from lipase degradation. Potential difference in phospholipids composition of large LDs may also contribute to the higher lipid storage capacity than that of small LDs (Cohen et al., 2015). The sizes and the volume of LDs are dependent on two major mechanisms: the growth activity of LDs that is dependent either on the activity of TAG synthesis or the atypical LD fusion that is mediated by CIDE proteins. Second, it is also dependent on the rate of TAG degradation that is controlled by cytosolic and lysosomal lipases. Growth by quick fusion of LDs through coalescence has also observed in some specific pharmacological conditions but its physiological relevance remains to be solved.

\section{LD GROWTH BY ELEVATED TAG SYNTHESIS}

As the core of LDs and the basic energy sources, the amount of TAG directly determines the size LDs and the amount of stored lipids. LD growth can occur by local synthesis of TAGs on LDs (Figure 2A), or by transferring TAG that is synthesized on ER to LDs. TAG is generated by a chain of biochemical reactions using FA as the substrate. Cells can either uptake FA from extracellular environment or generate FA by de novo synthesis. Various fatty acid transporting proteins are responsible for FA uptake and intracellular trafficking. De novo synthesis of FA is catalyzed by a series of enzymatic reactions and regulated by key transcriptional factors such as sterol regulatory element-binding protein-1c (SREBP-1c), liver X receptor (LXR), and carbohydrate response element binding protein (ChREBP) (Ferré and Foufelle, 2010; Strable and Ntambi, 2010).

TAG is synthesized by the addition of FA to glycerol-3 phosphate through the sequential enzymatic reactions catalyzed by glycerol-3-phosphate O-acyltransferase (GPAT), 1-acylglycerol-3-phosphate O-acyltransferase (AGPAT), phosphatidic acid phosphatase (PAP)/lipin, and diglyceride acyltransferase (DGAT). Most of these enzymes are localized on ER where TAG is generated and transported to LDs through unknown mechanism (Kory et al., 2016). Certain subset of enzymes (ACSL1, ACSL3, ACSL4, GPAT4, AGPAT3, Lipin1 and DGAT2) are reported to be specifically localized to LDs and promote local TAG synthesis and LD growth (Brasaemle et al., 2004; Fujimoto et al., 2007; Khelef 

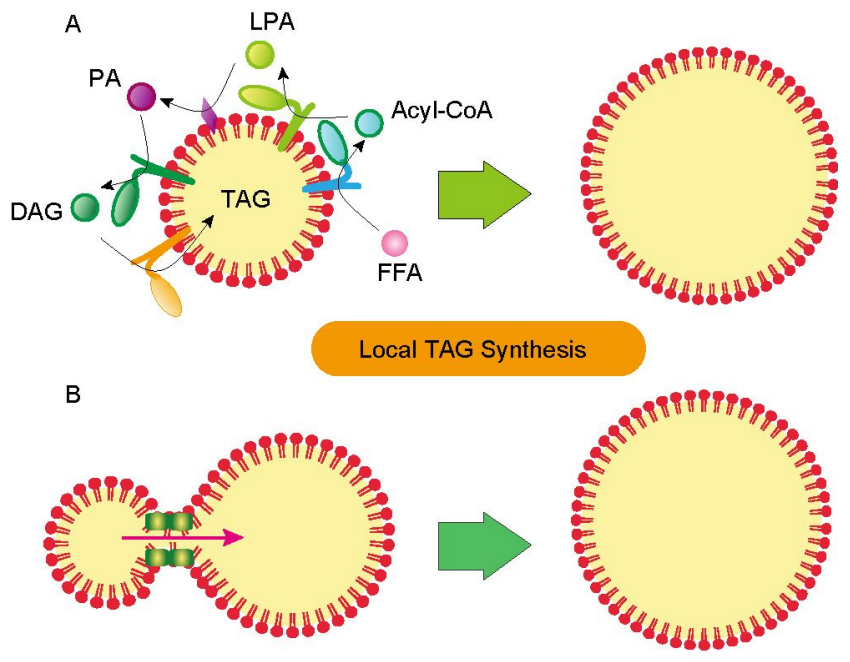

Atypical Fusion by Lipid Transfer

Figure 2 Two major mechanisms of LD growth. A, Many enzymes are localized to LD surface and TAG is synthesized locally to promoted LD growth. B, Atypical fusion of LDs mediated by CIDE proteins that promote LD growth and lipid storage. FFA, free fatty acid; LPA, lyso-phosphatidic acid; PA, phosphatidic acid; DAG, diacylglycerol; TAG, triacylglycerol.

et al., 1998; Kuerschner et al., 2008; Liu et al., 2004; Pol et al., 2014; Poppelreuther et al., 2012; Stone et al., 2009; Valdearcos et al., 2011; Wang et al., 2011; Wilfling et al., 2013). Growth of LD would also require phospholipids to stabilize the neutral lipid core. CTP:phosphocholine cytidylyltransferase (CCT), a rate-limiting enzyme in phospholipid synthesis pathway is responsible for the replenishment of phospholipids during the rapid growth of LDs. Newly synthesized PC may be transferred from ER to LDs by LD-ER conduit or other unknown pathways. LD growth through elevated TAG synthesis is a general mechanism for enhanced energy storage and probably reduced lipotocixity in many cell types from lower organisms such as yeast, C. elegans, fly to human (Murphy, 2012).

\section{LD GROWTH PROMOTED BY ATYPICAL FUSION}

Cells (adipocytes, hepatocytes, sebocytes etc.) that contain large LDs and have high capacity in storing lipids have adapted a special mechanism to efficiently increase lipid storage by special form of LD fusion that is mediated by CIDE proteins. Using genetically modified animal models, we have found that CIDE family proteins (including Cidea, Cideb, and Cidec/Fsp27) are important regulators of lipid storage in the adipocytes, hepatocytes, skin sebocytes and the mammary epithelia. The development of obesity and fatty liver diseases have been positively correlated with their protein levels (Matsusue et al., 2008; Toh et al., 2008; $\mathrm{Xu}$ et al., 2016). On the contrary, CIDEs deficiency leads to lipodystrophy, insulin resistance, dry skin and newborn death. In particular, white adipocytes require Cidec/Fsp27 for the formation of unilocular LD since its deficiency results in the accumulation of multilocular small LDs and markedly reduced lipid storage (Nishino et al., 2008; Rubio-Cabezas et al., 2009; Toh et al., 2008). Reduced LD sizes and low lipid storage is also observed in Cideb deficient hepatocytes, Cidea deficient skin sebocytes, brown adipocytes and mammary epithelia cells (Li et al., 2007; Wang et al., 2012; Wu et al., 2014b; Zhang et al., 2014; Zhou et al., 2003).

How do CIDE proteins promote LD growth and increase lipid storage? We have observed that these proteins are enriched and clustered at LD contact sites (LDCS) where they generate a potential pore (or channel structure) to allow lipid to exchange among contacted LD pair. The higher internal pressure in smaller LD will then drive the internal lipid transfer from the smaller LD to the large one to form a larger LD (Gong et al., 2011). As this process is different from SNARE-mediated membrane fusion, we define this as atypical LD fusion (Figure 3). We have further identified several proteins that interact with Cidec to promote LD fusion and unilocular LD formation in adipocytes. One of such protein is Plin1, a LD-associated protein that originally found to protect LD from lipase digestion. By interacting with the $\mathrm{N}$-terminal domain of Cidec, Plin1 maintains Cidec in a correct conformation and may stabilize the Cidec fusion complex and increase the efficiency of LD fusion (Sun et al., 2013). We also identified Rab8a as an important regulator of Cidec-mediated LD fusion. GTPase-activating proteins AS160 (Akt substrate

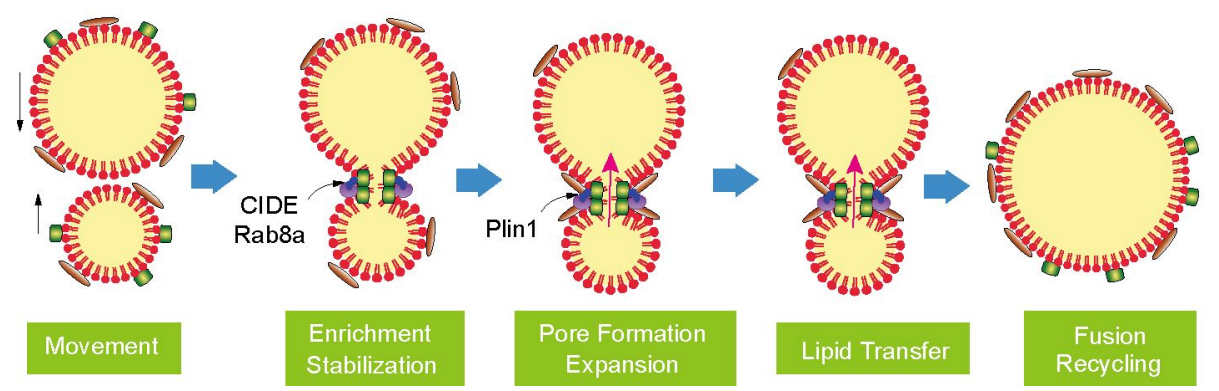

Figure 3 Model of CIDE-mediated atypical fusion of LD that includes LD movement; enrichment of CIDEs at LD contact site (LDCS) and stabilization of CIDE protein complex by Rab8a; formation of fusion pore that is stabilized by Plin1, and initiation of lipid transfer from smaller to larger LDs that is dependent on internal pressure and other unknown factors; and the final completion of LD fusion and redistribution or recycling of CIDE protein complex. 
of $160 \mathrm{kD}$ ) and guanine nucleotide exchange factor MSS4 (mammalian suppressor of Sec4) that modulate Rab8a activity, are also important in regulating Cidec-mediated atypical LD fusion (Wu et al., 2014a). In summary, CIDE-mediated atypical LD fusion and growth can be characterized into a few steps. First, LDs move quickly in the cell that may depend on microtubule. Second, LDs contact each other and form a tight junction with the enrichment of CIDE proteins at the contact site. This step is mediated by CIDE proteins and regulated by Rab8a. Third, fusion pore is formed and stabilized, and this step is likely dependent on Plin1 in adipocytes. Fourth, lipid transfer from smaller to larger LDs that is dependent on surface tension and internal pressure of contacted LD pairs. Finally, when fusion completed, the fusion protein complex is disassembled and CIDE proteins and its associated proteins are redistributed on the surface of LDs (Figure 3 ). In the future, it will be interesting to identify novel factors in CIDE-mediated LD fusion, characterize the structure of the fusion complex and delineate the regulatory pathways that mediate nutrient and hormonal response in CIDE-mediated atypical LD fusion.

\section{THE SHRINKAGE AND DEGRADATION OF LDS}

Another important mechanism to regulate lipid storage and LD sizes is the degradation of TAG in the neutral lipid core by lipases to generate FFAs for providing energy substrate during increased energy demand (endurance exercise) and energy deprivation (fasting or starve). FFAs released from TAG degradation are transported intracellularly to mitochondria for oxidation and ATP generation. FFAs released from WAT enter into the circulation, and uptaken and transported by various FA binding/transporting proteins for further oxidation in muscle, heart, liver and other tissues. Glycerol, another production of TAG degradation, is released to the circulation and converted to glucose in the liver (Large et al., 2004; Zechner et al., 2009). There are two well-studied TAG hydrolysis pathways. First is the direct degradation of TAG by neutral lipases that binds to LDs. Another is the degradation of TAG in LDs by lysosomal acid lipase (LAL) that is localized to lysosomes. This pathway requires the delivery of LDs to lysosome by autophagosomes (Schweiger and Zechner, 2015; Singh et al., 2009).

\section{LD DEGRADATION BY NEUTRAL LIPASES}

Neutral cytosolic lipases responsible for TAG degradation include adipose triglyceride lipase (ATGL), hormone sensitive lipase (HSL), and monoglyceride lipase (MGL). These enzymes work step-wise to hydrolyze TAGs from LDs. ATGL catalyzes the first step of lipolysis by hydrolyzing
TAG and generating FFA and DAG, DAG is successively hydrolyzed by HSL to liberate FFA and monoacylglycerol (MAG). Finally, MGL converts MAG to glycerol and FFA. ATGL has been shown to play a crucial role in animal survival, organ function, as well as cancer cell growth, and cachexia (Haemmerle et al., 2006; Zechner, 2015). Human mutation of ATGL also results in neutral lipid storage disease with myopathy (Fischer et al., 2007).

Neutral lipase-mediated LD degradation is a complex, multi-factorial process that is regulated by hormones, cytokines, adipokines and protein-protein interaction (Zechner, 2015; Zechner et al., 2009). The catalytic activity of ATGL is modified by its interaction with other proteins including abhydrolase domain containing 5 (ABHD5/CGI-58) and G0/G1 switch gene 2 (G0S2). Interaction with CGI-58 significantly increases the hydrolase activity of ATGL. In contrast, when ATGL interacts with G0S2, its enzymatic activity is strongly inhibited ( $\mathrm{Lu}$ et al., 2010; Yang et al., 2010). Under basal condition, Plin 1 forms a protective barrier on LDs, preventing lipases from accessing LDs (Brasaemle et al., 2000; Martinez-Botas et al., 2000; Tansey et al., 2001). Plin2 and Plin5 also play similar roles to that of Plin1 (Kimmel and Sztalryd, 2016; Mason et al., 2014; Mason and Watt, 2015; Sztalryd and Kimmel, 2014). In addition, Plin1 interacts with CGI-58, rendering low ATGL activity in hydrolyze TAG (Lass et al., 2006; Yamaguchi et al., 2004). HSL is predominantly present in the cytosol and does not appear to contribute to basal lipolysis (Miyoshi et al., 2008) (Figure 4A). MGL hydrolyzes MAG that is produced by ATGL and HSL to generate FFAs and glycerol. One interesting feature of MGL is that is has no significant substrate specificity and can be localized to LDs, ER, cytoplasm, and the plasma membrane. The activity of ATGL and MGL is also important in cancer pathogenesis (Nomura et al., 2010; Zechner, 2015).

Catecholamines (CA) acting on $\beta$-adrenergic receptors $(\beta-A R)$ is the most potent stimulatory signals for lipolysis (Lafontan and Berlan, 1993). Activation of $\beta$-AR by $\mathrm{CA}$ leads to the activation of adenylate cyclase, increased cAMP levels, and subsequent activation of cAMP-dependent protein kinase-A (PKA) (Collins et al., 2004; Holm, 2003) (Figure 4B). The most well studied PKA substrates in lipolysis are Plin1 and HSL. When Plin1 is phosphorylated by PKA, CGI-58 is released from Plin1-CGI-58 complex, leading to the association between CGI-58 and ATGL, and high TAG hydrolysis activity (Gandotra et al., 2011; Miyoshi et al., 2008; Smirnova et al., 2006; Watt and Steinberg, 2008; Zimmermann et al., 2004) (Figure 4B). Plin5 is also a downstream target of PKA and may play a similar role in hormone-stimulated lipolysis to that of Plin1 (Macpherson et al., 2013; Pollak et al., 2015). Phosphorylated Plin1 also recruit phosphorylated HSL to LDs and modulate its activity 


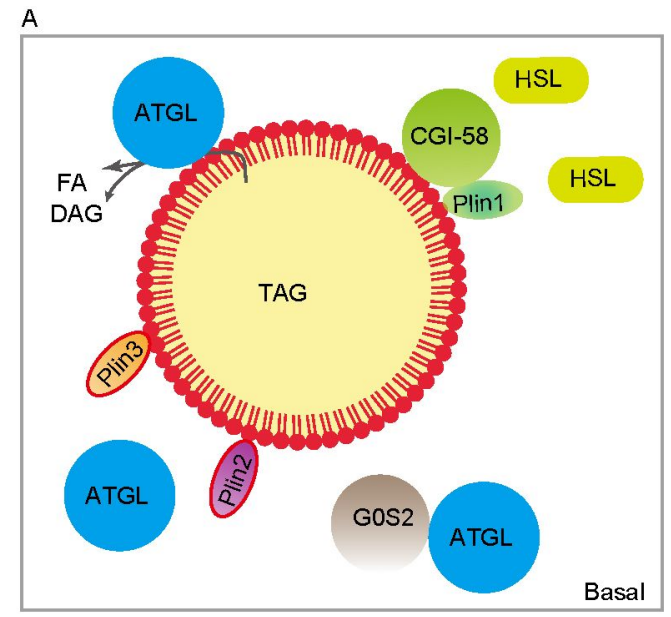

B
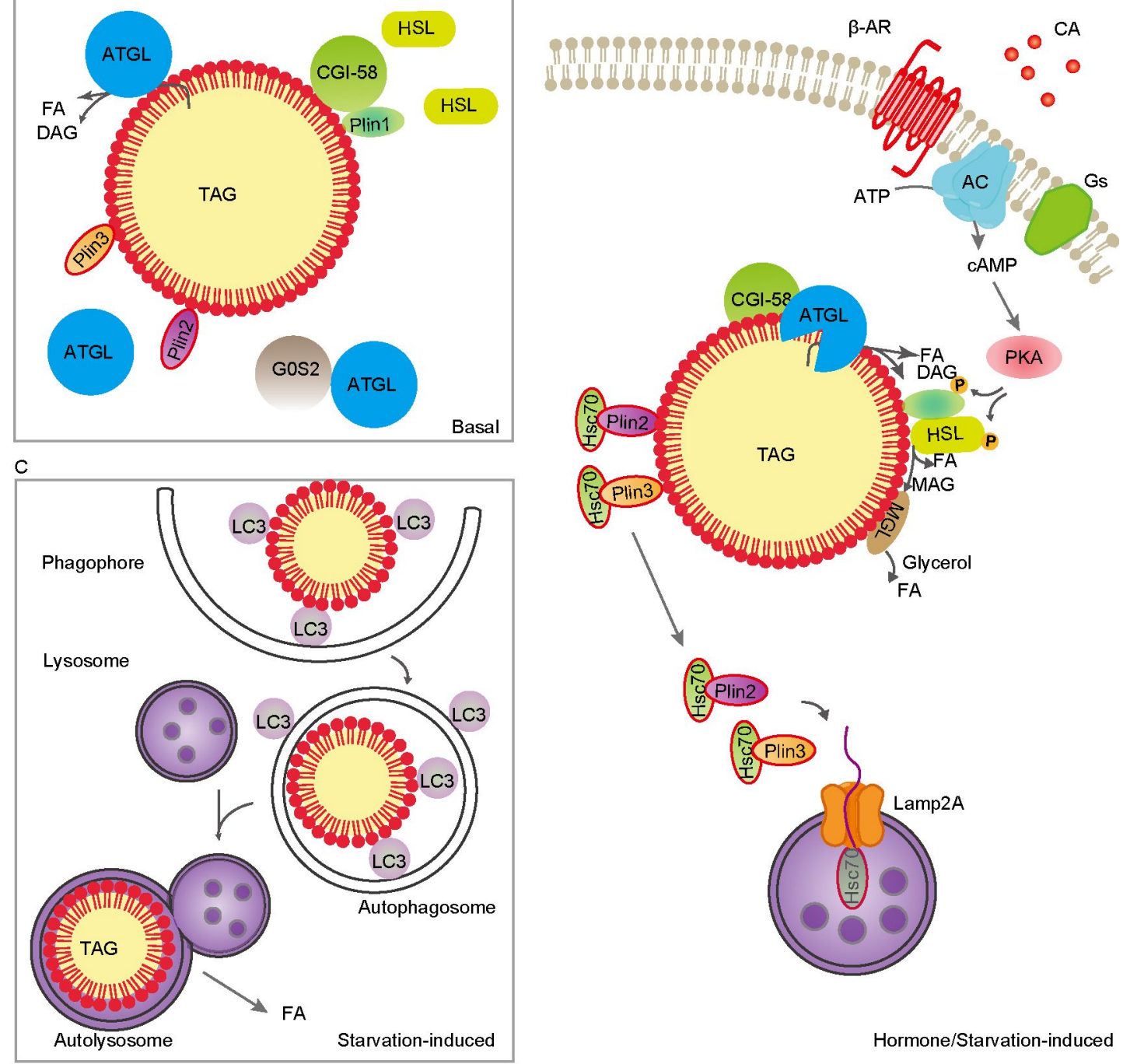

Hormone/Starvation-induced

Figure 4 The regulation of LD degradation by cytosolic lipases under basal (A), hormone or starvation conditions (B) or LD degradation by lysosomal lipases under starvation condition (C). CA, catecholamine; $\beta$-AR, $\beta$-adrenergic receptor; Gs, stimulative regulative G-protein.

(Miyoshi et al., 2006; Wang et al., 2009; Watt et al., 2006). However, the physiological relevance of the post translational modification of ATGL in controlling lipolysis and lipid storage remains to be controversial. Recent analysis has revealed that chaperone-mediated autophagy (CMA) may cross talk with neutral lipase-mediated lipolysis by removing Plin2 and Plin3 from LD surface and triggering their degradation by lysosomes through their association with chaperone Hsc70, resulting in the translocation of ATGL to LD for TAG degradation (Kaushik and Cuervo, 2015) (Figure 4B).

\section{LD DEGRADATION MEDIATED BY LYSOSOMAL ACIDIC LIPASES}

For a long time, lysosomal acidic lipases (LALs) are believed to be responsible for the degradation of lipid membranes and exogenous lipoproteins that are taken up by cells and deliv- ered to lysosome. In 2009, Singh and colleagues found that under prolonged fasting condition, autophagy plays an important role in LD degradation by selective uptake and sequestering of LDs to autophagosome to deliver to lysosome for degradation (lipophagy) in the liver (Singh et al., 2009). Since then, lipohagy has been discovered in many cell types as well as lower organisms such as yeast and fungi (Wang, 2016). The precise mechanism of lipophagy activation in particular how autophagosome specifically recognizes LDs is not clear and remains a hot debate. As LC3-I, LC3-II and several regulatory proteins in autophagic pathways are found to be enriched in LDs, these proteins may play a role in lipophagy activation (Singh et al., 2009) (Figure 4C). Rab7, a small GTPase, has also shown to associate with LDs in starved hepatocytes and may be important for autophagy-mediated LD degradation (Schroeder et al., 2015). In addition, crosstalk between autophagic- and neutral lipase-mediate LD degrada- 
tion has been demonstrated as PNPLA5, a lipase that is localized to LDs, is required for efficient autophagy by likely supplying FAs for phospholipid production during autophagic progression (Dupont et al., 2014).

\section{OTHER IMPORTANT FACTORS CONTROLLING LD SIZES AND LIPID STORAGE}

In addition to the above mentioned LD growth and degradation pathways, the sizes of LDs and the intracellular lipid storage capacity are regulated by several other factors including Seipin, Pnpla3, Hsd17b13, Cavin and Ces1 (Bi et al., 2014; Cai et al., 2015; Cartwright et al., 2015; Grippa et al., 2015; Han et al., 2015; Romeo et al., 2008; Su et al., 2014; Szymanski et al., 2007; Wang et al., 2016; Wolinski et al., 2015). Seipin is originally discovered due to its mutation in severe congenital generalized lipodystrophy (Magré et al., 2001). Fld1, a homolog of Seipin in yeast, controls LD sizes as its deficiency results in the accumulation of "supersized" LDs (Fei et al., 2008; Szymanski et al., 2007). Seipin homolog in fly also plays a conserved role in controlling lipid storage and LD sizes (Tian et al., 2011). Seipin is an ER-associated protein that may function to control localized PA production, $\mathrm{Ca}^{2+}$ homeostasis as well as mediate ER-LD contact (Bi et al., 2014; Cartwright and Goodman, 2012; Grippa et al., 2015; Han et al., 2015; Wolinski et al., 2015). Most recent research by Wang, et al. has indicated that Seipin forms discrete foci on ER to control nascent LD growth (Wang et al., 2016). Genetic or proteomic screening of human fatty liver patients leads to the identification of Pnpla3 and Hsd17b13 that are correlated with the development of liver steatosis (Chamoun et al., 2013; Romeo et al., 2008; Su et al., 2014). Interestingly, adipocyte is one of the most caveolae-enriched cell types and loss of caveolae leads to lipodystrophic phenotype in humans. Recent analysis shows that caveolae may participate in adipocyte response to mechanotension and lipid store fluctuation (Dwianingsih et al., 2010; Shastry et al., 2010). Regulatory factors such as PPARgamma and AKT can also regulate lipolysis and lipid storage in adipocytes (Krahmer et al., 2013).

\section{CONCLUSION AND FUTURE PERSPECTIVES}

Sustainable energy supply is critical for survival under adverse conditions such as fasting, prolonged exercise and stress. On the contrary, excess energy storage due to increased energy uptake and reduced energy expenditure results in the development of metabolic disorders including obesity, diabetes and fatty liver disease. LDs are the subcellular organelles responsible for energy storage. The sizes and number of LDs reflect the energy storage capacity and are regulated by many biological processes including localized
TAG synthesis, atypical LD fusion and LD degradation mediated by neutral lipases and autophagy mediated lysosomal acidic lipases. Coordinated regulation of lipid metabolism, glucose metabolism, mitochondrial activity, FA oxidation, ER and Golgi activity are also important in regulating lipid storage. In addition, nutrient response, hormonal stimulation and other environmental factors may also contribute to the intracellular LD sizes and lipid storage.

Therapeutical strategies targeting to lipid storage pathway in controlling the development of metabolic disorders were initiated many years ago and significant progress have been made in the identification of compounds or molecules that control the activation of metabolic consumption of lipids by increasing mitochondrial FA oxidaiton, reducing lipid uptake and synthesis and enhancing lipase activity. Our in-depth understanding of the molecular mechanism of lipid storage regulation would provide novel therapeutical approaches for the development of effective tools for combating lipid storage related diseases.

Compliance and ethics The author(s) declare that they have no conflict of interest.

Acknowledgements This work was supported by the National Natural Science Foundation of China (31420040, 31321003 to Peng Li, 31501089 to Jinhai Yu), the National Basic Research Program (2013CB530602 to Peng Li), and the China Postdoctoral Science Foundation (2015M581079 to Jinhai $Y u)$.

Anand, P., Cermelli, S., Li, Z., Kassan, A., Bosch, M., Sigua, R., Huang, L., Ouellette, A.J., Pol, A., Welte, M.A., and Gross, S.P. (2012). A novel role for lipid droplets in the organismal antibacterial response. eLife 1, e00003.

Baenke, F., Peck, B., Miess, H., and Schulze, A. (2013). Hooked on fat: the role of lipid synthesis in cancer metabolism and tumour development. Dis Model Mech 6, 1353-1363.

Barbosa, A.D., Savage, D.B., and Siniossoglou, S. (2015). Lipid dropletorganelle interactions: emerging roles in lipid metabolism. Curr Opin Cell Biol 35, 91-97.

Bartz, R., Li, W.H., Venables, B., Zehmer, J.K., Roth, M.R., Welti, R., Anderson, R.G.W., Liu, P., and Chapman, K.D. (2007). Lipidomics reveals that adiposomes store ether lipids and mediate phospholipid traffic. J Lipid Res 48, 837-847.

Beller, M., Thiel, K., Thul, P.J., and Jäckle, H. (2010). Lipid droplets: a dynamic organelle moves into focus. FEBS Lett 584, 2176-2182.

Bi, J., Wang, W., Liu, Z., Huang, X., Jiang, Q., Liu, G., Wang, Y., and Huang, $X$. (2014). Seipin promotes adipose tissue fat storage through the ER $\mathrm{Ca}^{2+}$-ATPase SERCA. Cell Metab 19, 861-871.

Bindlish, S., Presswala, L.S., and Schwartz, F. (2015). Lipodystrophy: syndrome of severe insulin resistance. Postgrad Med 127, 511-516.

Brasaemle, D.L., Dolios, G., Shapiro, L., and Wang, R. (2004). Proteomic analysis of proteins associated with lipid droplets of basal and lipolytically stimulated 3T3-L1 adipocytes. J Biol Chem 279, 46835-46842.

Brasaemle, D.L., Rubin, B., Harten, I.A., Gruia-Gray, J., Kimmel, A.R., and Londos, C. (2000). Perilipin A increases triacylglycerol storage by decreasing the rate of triacylglycerol hydrolysis. J Biol Chem 275, 38486-38493.

Cai, Y., Goodman, J.M., Pyc, M., Mullen, R.T., Dyer, J.M., and Chapman, K.D. (2015). Arabidopsis SEIPIN proteins modulate triacylglycerol accumulation and influence lipid droplet proliferation. Plant Cell 
27, 2616-2636.

Camus, G., Vogt, D.A., Kondratowicz, A.S., and Ott, M. (2013). Lipid droplets and viral infections. Methods Cell Biol 116, 167-190.

Cartwright, B.R., Binns, D.D., Hilton, C.L., Han, S., Gao, Q., and Goodman, J.M. (2015). Seipin performs dissectible functions in promoting lipid droplet biogenesis and regulating droplet morphology. Mol Biol Cell 26, 726-739.

Cartwright, B.R., and Goodman, J.M. (2012). Seipin: from human disease to molecular mechanism. J Lipid Res 53, 1042-1055.

Chamoun, Z., Vacca, F., Parton, R.G., and Gruenberg, J. (2013). PNPLA3/adiponutrin functions in lipid droplet formation. Biol Cell 105, 219-233.

Chen, Y., and Li, P. (2016). Fatty acid metabolism and cancer development. Sci Bull 61, 1473-1479.

Cohen, B.C., Shamay, A., and Argov-Argaman, N. (2015). Regulation of lipid droplet size in mammary epithelial cells by remodeling of membrane lipid composition-A potential mechanism. PLoS ONE 10, e0121645.

Collins, S., Cao, W., and Robidoux, J. (2004). Learning new tricks from old dogs: $\beta$-adrenergic receptors teach new lessons on firing up adipose tissue metabolism. Mol Endocrinol 18, 2123-2131.

Crawford, S.E., and Desselberger, U. (2016). Lipid droplets form complexes with viroplasms and are crucial for rotavirus replication. Curr Opin Virol 19, 11-15.

D’Avila, H., Maya-Monteiro, C.M., and Bozza, P.T. (2008). Lipid bodies in innate immune response to bacterial and parasite infections. Int Immunopharmacol 8, 1308-1315.

Dichlberger, A., Schlager, S., Maaninka, K., Schneider, W.J., and Kovanen, P.T. (2014). Adipose triglyceride lipase regulates eicosanoid production in activated human mast cells. J Lipid Res 55, 2471-2478.

Dupont, N., Chauhan, S., Arko-Mensah, J., Castillo, E.F., Masedunskas, A., Weigert, R., Robenek, H., Proikas-Cezanne, T., and Deretic, V. (2014). Neutral lipid stores and lipase PNPLA5 contribute to autophagosome biogenesis. Curr Biol 24, 609-620.

Dwianingsih, E.K., Takeshima, Y., Itoh, K., Yamauchi, Y., Awano, H., Malueka, R.G., Nishida, A., Ota, M., Yagi, M., and Matsuo, M. (2010). A Japanese child with asymptomatic elevation of serum creatine kinase shows PTRF-CAVIN mutation matching with congenital generalized lipodystrophy type 4. Mol Genets Metab 101, 233-237.

Farese, R.V.Jr., and Walther, T.C. (2016). Lipid droplets go nuclear. J Cell Biol 212, 7-8.

Fei, W., Shui, G., Gaeta, B., Du, X., Kuerschner, L., Li, P., Brown, A.J., Wenk, M.R., Parton, R.G., and Yang, H. (2008). Fld1p, a functional homologue of human seipin, regulates the size of lipid droplets in yeast. J Cell Biol 180, 473-482.

Ferré, P., and Foufelle, F. (2010). Hepatic steatosis: a role for de novo lipogenesis and the transcription factor SREBP-1c. Diabetes Obes Metab 12, 83-92.

Filipe, A., and McLauchlan, J. (2015). Hepatitis C virus and lipid droplets: finding a niche. Trends Mol Med 21, 34-42.

Fischer, J., Lefèvre, C., Morava, E., Mussini, J.M., Laforêt, P., Negre-Salvayre, A., Lathrop, M., and Salvayre, R. (2007). The gene encoding adipose triglyceride lipase (PNPLA2) is mutated in neutral lipid storage disease with myopathy. Nat Genet 39, 28-30.

Frayn, K.N. (2001). Adipose tissue and the insulin resistance syndrome. Proc Nutr Soc 60, 375-380.

Fujimoto, T., and Parton, R.G. (2011). Not just fat: the structure and function of the lipid droplet. Cold Spring Harb Perspect Biol 3, a004838-a004838.

Fujimoto, Y., Itabe, H., Kinoshita, T., Homma, K.J., Onoduka, J., Mori, M., Yamaguchi, S., Makita, M., Higashi, Y., Yamashita, A., and Takano, T. (2007). Involvement of ACSL in local synthesis of neutral lipids in cytoplasmic lipid droplets in human hepatocyte HuH7. J Lipid Res 48, 1280-1292.

Gandotra, S., Lim, K., Girousse, A., Saudek, V., O’Rahilly, S., and Savage, D.B. (2011). Human frame shift mutations affecting the carboxyl terminus of perilipin increase lipolysis by failing to sequester the adipose triglyceride lipase (ATGL) coactivator AB-hydrolase-containing 5 (ABHD5). J Biol Chem 286, 34998-35006.

Gao, Q., and Goodman, J.M. (2015). The lipid droplet - a well-connected organelle. Front Cell Dev Biol 3, 49.

Gong, J., Sun, Z., and Li, P. (2009). CIDE proteins and metabolic disorders. Curr Opin Lipidol 20, 121-126.

Gong, J., Sun, Z., Wu, L., Xu, W., Schieber, N., Xu, D., Shui, G., Yang, H., Parton, R.G., and Li, P. (2011). Fsp27 promotes lipid droplet growth by lipid exchange and transfer at lipid droplet contact sites. J Cell Biol 195, 953-963.

Grippa, A., Buxó, L., Mora, G., Funaya, C., Idrissi, F.Z., Mancuso, F., Gomez, R., Muntanyà, J., Sabidó, E., and Carvalho, P. (2015). The seipin complex Fld1/Ldb16 stabilizes ER-lipid droplet contact sites. J Cell Biol 211, 829-844.

Gross, D.A., and Silver, D.L. (2014). Cytosolic lipid droplets: from mechanisms of fat storage to disease. Crit Rev Biochem Mol Biol 49, 304-326.

Grundy, S.M. (2015). Adipose tissue and metabolic syndrome: too much, too little or neither. Eur J Clin Invest 45, 1209-1217.

Haemmerle, G., Lass, A., Zimmermann, R., Gorkiewicz, G., Meyer, C., Rozman, J., Heldmaier, G., Maier, R., Theussl, C., Eder, S., Kratky, D., Wagner, E.F., Klingenspor, M., Hoefler, G., and Zechner, R. (2006). Defective lipolysis and altered energy metabolism in mice lacking adipose triglyceride lipase. Science 312, 734-737.

Han, S., Binns, D.D., Chang, Y.F., and Goodman, J.M. (2015). Dissecting seipin function: the localized accumulation of phosphatidic acid at $\mathrm{ER} / \mathrm{LD}$ junctions in the absence of seipin is suppressed by Seil $\mathrm{p} \Delta \mathrm{Nterm}$ only in combination with Ldb16p. BMC Cell Biol 16, 29.

Herman, I.P. (2016). Physics of the Human Body. (Berlin: Springer).

Hinson, E.R., and Cresswell, P. (2009). The antiviral protein, viperin, localizes to lipid droplets via its N-terminal amphipathic $\alpha$-helix. Proc Natl Acad Sci USA 106, 20452-20457.

Holm, C. (2003). Molecular mechanisms regulating hormone-sensitive lipase and lipolysis. Biochm Soc Trans 31, 1120-1124.

Jacquier, N., Choudhary, V., Mari, M., Toulmay, A., Reggiori, F., and Schneiter, R. (2011). Lipid droplets are functionally connected to the endoplasmic reticulum in Saccharomyces cerevisiae. J Cell Sci 124, 2424-2437.

Kassan, A., Herms, A., Fernández-Vidal, A., Bosch, M., Schieber, N.L., Reddy, B.J.N., Fajardo, A., Gelabert-Baldrich, M., Tebar, F., Enrich, C., Gross, S.P., Parton, R.G., and Pol, A. (2013). Acyl-CoA synthetase 3 promotes lipid droplet biogenesis in ER microdomains. J Cell Biol 203, 985-1001.

Kaushik, S., and Cuervo, A.M. (2015). Degradation of lipid droplet-associated proteins by chaperone-mediated autophagy facilitates lipolysis. Nat Cell Biol 17, 759-770.

Khelef, N., Buton, X., Beatini, N., Wang, H., Meiner, V., Chang, T.Y., Farese, R.V., Maxfield, F.R., and Tabas, I. (1998). Immunolocalization of acylcoenzyme A:cholesterol O-acyltransferase in macrophages. J Biol Chem 273, 11218-11224.

Kimmel, A.R., and Sztalryd, C. (2016). The perilipins: major cytosolic lipid droplet-associated proteins and their roles in cellular lipid storage, mobilization, and systemic homeostasis. Annu Rev Nutr 36, 471-509.

Knoblach, B., and Rachubinski, R.A. (2015). Transport and retention mechanisms govern lipid droplet inheritance in Saccharomyces cerevisiae. Traffic 16, 298-309.

Kory, N., Farese, R.V., Jr., and Walther, T.C. (2016). Targeting fat: mechanisms of protein localization to lipid droplets. Trends Cell Biol 26, 535-546.

Krahmer, N., Farese Jr, R.V., and Walther, T.C. (2013). Balancing the fat: lipid droplets and human disease. EMBO Mol Med 5, 973-983.

Kuerschner, L., Moessinger, C., and Thiele, C. (2008). Imaging of lipid biosynthesis: how a neutral lipid enters lipid droplets. Traffic 9, 338-352.

Lafontan, M., and Berlan, M. (1993). Fat cell adrenergic receptors and the control of white and brown fat cell function. J Lipid Res 34, 1057-1091. 
Large, V., Peroni, O., Letexier, D., Ray, H., and Beylot, M. (2004). Metabolism of lipids in human white adipocyte. Diabetes Metab 30, 294-309.

Lass, A., Zimmermann, R., Haemmerle, G., Riederer, M., Schoiswohl, G., Schweiger, M., Kienesberger, P., Strauss, J.G., Gorkiewicz, G., and Zechner, R. (2006). Adipose triglyceride lipase-mediated lipolysis of cellular fat stores is activated by CGI-58 and defective in Chanarin-Dorfman Syndrome. Cell Metab 3, 309-319.

Li, J.Z., Ye, J., Xue, B., Qi, J., Zhang, J., Zhou, Z., Li, Q., Wen, Z., and Li, P. (2007). Cideb regulates diet-induced obesity, liver steatosis, and insulin sensitivity by controlling lipogenesis and fatty acid oxidation. Diabetes 56, 2523-2532.

Liu, L., Zhang, K., Sandoval, H., Yamamoto, S., Jaiswal, M., Sanz, E., Li, Z., Hui, J., Graham, B.H., Quintana, A., and Bellen, H.J. (2015). Glial lipid droplets and ROS induced by mitochondrial defects promote neurodegeneration. Cell 160, 177-190.

Liu, P., Bartz, R., Zehmer, J.K., Ying, Y., Zhu, M., Serrero, G., and Anderson, R.G.W. (2007). Rab-regulated interaction of early endosomes with lipid droplets. Biochim Biophys Acta 1773, 784-793.

Liu, P., Ying, Y., Zhao, Y., Mundy, D.I., Zhu, M., and Anderson, R.G.W. (2004). Chinese hamster ovary K2 cell lipid droplets appear to be metabolic organelles involved in membrane traffic. J Biol Chem 279, 3787-3792.

Lodhi, I.J., and Semenkovich, C.F. (2014). Peroxisomes: a nexus for lipid metabolism and cellular signaling. Cell Metab 19, 380-392.

Lu, X., Yang, X., and Liu, J. (2010). Differential control of ATGL-mediated lipid droplet degradation by CGI-58 and G0S2. Cell Cycle 9, 2791-2797.

Macpherson, R.E.K., Vandenboom, R., Roy, B.D., and Peters, S.J. (2013). Skeletal muscle PLIN3 and PLIN5 are serine phosphorylated at rest and following lipolysis during adrenergic or contractile stimulation. Physiol Rep 1, e00084.

Magré, J., Delépine, M., Khallouf, E., Gedde-Dahl, T., Van Maldergem, L., Sobel, E., Papp, J., Meier, M., Mégarbané, A., Bachy, A., Verloes, A., d'Abronzo, F.H., Seemanova, E., Assan, R., Baudic, N., Bourut, C., Czernichow, P., Huet, F., Grigorescu, F., de Kerdanet, M., Lacombe, D., Labrune, P., Lanza, M., Loret, H., Matsuda, F., Navarro, J., Nivelon-Chevalier, A., Polak, M., Robert, J.J., Tric, P., Tubiana-Rufi, N., Vigouroux, C., Weissenbach, J., Savasta, S., Maassen, J.A., Trygstad, O., Bogalho, P., Freitas, P., Medina, J.L., Bonnicci, F., Joffe, B.I., Loyson, G., Panz, V.R., Raal, F.J., O’Rahilly, S., Stephenson, T., Kahn, C.R., Lathrop, M., Capeau, J., and Capeau, J. (2001). Identification of the gene altered in Berardinelli-Seip congenital lipodystrophy on chromosome 11q13. Nat Genet 28, 365-370.

Martinez-Botas, J., Anderson, J.B., Tessier, D., Lapillonne, A., Chang, B.H.J., Quast, M.J., Gorenstein, D., Chen, K.H., and Chan, L. (2000). Absence of perilipin results in leanness and reverses obesity in Lepr(db/db) mice. Nat Genet 26, 474-479.

Mason, R.R., Mokhtar, R., Matzaris, M., Selathurai, A., Kowalski, G.M., Mokbel, N., Meikle, P.J., Bruce, C.R., and Watt, M.J. (2014). PLIN5 deletion remodels intracellular lipid composition and causes insulin resistance in muscle. Mol Metab 3, 652-663.

Mason, R.R., and Watt, M.J. (2015). Unraveling the roles of PLIN5: linking cell biology to physiology. Trends Endocrinol Metab 26, 144-152.

Matsusue, K., Kusakabe, T., Noguchi, T., Takiguchi, S., Suzuki, T., Yamano, S., and Gonzalez, F.J. (2008). Hepatic steatosis in leptin-deficient mice is promoted by the PPAR $\gamma$ target gene Fsp27. Cell Metab 7, 302-311.

Melo, R.C.N., and Weller, P.F. (2016). Lipid droplets in leukocytes: organelles linked to inflammatory responses. Exp Cell Res 340, 193-197.

Miyoshi, H., Perfield Ii, J.W., Obin, M.S., and Greenberg, A.S. (2008). Adipose triglyceride lipase regulates basal lipolysis and lipid droplet size in adipocytes. J Cell Biochem 105, 1430-1436.

Miyoshi, H., Souza, S.C., Zhang, H.H., Strissel, K.J., Christoffolete, M.A., Kovsan, J., Rudich, A., Kraemer, F.B., Bianco, A.C., Obin, M.S., and Greenberg, A.S. (2006). Perilipin promotes hormone-sensitive lipasemediated adipocyte lipolysis via phosphorylation-dependent and -independent mechanisms. J Biol Chem 281, 15837-15844.

Murphy, D.J. (2012). The dynamic roles of intracellular lipid droplets: from archaea to mammals. Protoplasma 249, 541-585.

Murphy, S., Martin, S., and Parton, R.G. (2009). Lipid droplet-organelle interactions; sharing the fats. Biochim Biophys Acta 1791, 441-447.

Nishino, N., Tamori, Y., Tateya, S., Kawaguchi, T., Shibakusa, T., Mizunoya, W., Inoue, K., Kitazawa, R., Kitazawa, S., Matsuki, Y., Hiramatsu, R., Masubuchi, S., Omachi, A., Kimura, K., Saito, M., Amo, T., Ohta, S., Yamaguchi, T., Osumi, T., Cheng, J., Fujimoto, T., Nakao, H., Nakao, K., Aiba, A., Okamura, H., Fushiki, T., and Kasuga, M. (2008). FSP27 contributes to efficient energy storage in murine white adipocytes by promoting the formation of unilocular lipid droplets. J Clin Invest 118, 2808.

Nomura, D.K., Long, J.Z., Niessen, S., Hoover, H.S., Ng, S.W., and Cravatt, B.F. (2010). Monoacylglycerol lipase regulates a fatty acid network that promotes cancer pathogenesis. Cell 140, 49-61.

Ohsaki, Y., Suzuki, M., and Fujimoto, T. (2014). Open questions in lipid droplet biology. Chem Biol 21, 86-96.

Pol, A., Gross, S.P., and Parton, R.G. (2014). Biogenesis of the multifunctional lipid droplet: lipids, proteins, and sites. J Cell Biol 204, 635-646.

Pollak, N.M., Jaeger, D., Kolleritsch, S., Zimmermann, R., Zechner, R., Lass, A., and Haemmerle, G. (2015). The interplay of protein kinase A and perilipin 5 regulates cardiac lipolysis. J Biol Chem 290, 1295-1306.

Poppelreuther, M., Rudolph, B., Du, C., Grossmann, R., Becker, M., Thiele, C., Ehehalt, R., and Fullekrug, J. (2012). The N-terminal region of acylCoA synthetase 3 is essential for both the localization on lipid droplets and the function in fatty acid uptake. J Lipid Res 53, 888-900.

Pu, J., Ha, C.W., Zhang, S., Jung, J.P., Huh, W.K., and Liu, P. (2011). Interactomic study on interaction between lipid droplets and mitochondria. Protein Cell 2, 487-496.

Qiu, B., Ackerman, D., Sanchez, D.J., Li, B., Ochocki, J.D., Grazioli, A., Bobrovnikova-Marjon, E., Diehl, J.A., Keith, B., and Simon, M.C. (2015). HIF2 $\alpha$-dependent lipid storage promotes endoplasmic reticulum homeostasis in clear-cell renal cell carcinoma. Cancer Discov 5, 652-667.

Rambold, A.S., Cohen, S., and Lippincott-Schwartz, J. (2015). Fatty acid trafficking in starved cells: regulation by lipid droplet lipolysis, autophagy, and mitochondrial fusion dynamics. Dev Cell 32, 678-692.

Robenek, H., Buers, I., Hofnagel, O., Robenek, M.J., Troyer, D., and Severs, N.J. (2009). Compartmentalization of proteins in lipid droplet biogenesis. Biochim Biophys Acta 1791, 408-418.

Romeo, S., Kozlitina, J., Xing, C., Pertsemlidis, A., Cox, D., Pennacchio, L.A., Boerwinkle, E., Cohen, J.C., and Hobbs, H.H. (2008). Genetic variation in PNPLA3 confers susceptibility to nonalcoholic fatty liver disease. Nat Genet 40, 1461-1465.

Rubio-Cabezas, O., Puri, V., Murano, I., Saudek, V., Semple, R.K., Dash, S., Hyden, C.S.S., Bottomley, W., Vigouroux, C., Magré, J., Raymond-Barker, P., Murgatroyd, P.R., Chawla, A., Skepper, J.N., Chatterjee, V.K., Suliman, S., Patch, A.M., Agarwal, A.K., Garg, A., Barroso, I., Cinti, S., Czech, M.P., Argente, J., O’Rahilly, S., Savage, D.B., and Savage, D.B. (2009). Partial lipodystrophy and insulin resistant diabetes in a patient with a homozygous nonsense mutation in CIDEC. EMBO Mol Med 1, 280-287.

Rutkowski, J.M., Stern, J.H., and Scherer, P.E. (2015). The cell biology of fat expansion. J Cell Biol 208, 501-512.

Saka, H.A., and Valdivia, R. (2012). Emerging roles for lipid droplets in immunity and host-pathogen interactions. Annu Rev Cell Dev Biol 28, 411-437.

Schrader, M. (2001). Tubulo-reticular clusters of peroxisomes in living COS-7 cells: dynamic behavior and association with lipid droplets. J Histochem Cytochem 49, 1421-1429.

Schroeder, B., Schulze, R.J., Weller, S.G., Sletten, A.C., Casey, C.A., and McNiven, M.A. (2015). The small GTPase Rab7 as a central regulator of hepatocellular lipophagy. Hepatology 61, 1896-1907.

Schweiger, M., and Zechner, R. (2015). Breaking the barrier-chaperonemediated autophagy of perilipins regulates the lipolytic degradation of fat. Cell Metab 22, 60-61.

Sell, H., Habich, C., and Eckel, J. (2012). Adaptive immunity in obesity and insulin resistance. Nat Rev Endocrinol 8, 709-716.

Shastry, S., Delgado, M.R., Dirik, E., Turkmen, M., Agarwal, A.K., and 
Garg, A. (2010). Congenital generalized lipodystrophy, type 4 (CGL4) associated with myopathy due to novel PTRF mutations. Am J Med Genet 152A, 2245-2253.

Singh, R., Kaushik, S., Wang, Y., Xiang, Y., Novak, I., Komatsu, M., Tanaka, K., Cuervo, A.M., and Czaja, M.J. (2009). Autophagy regulates lipid metabolism. Nature 458, 1131-1135.

Smirnova, E., Goldberg, E.B., Makarova, K.S., Lin, L., Brown, W.J., and Jackson, C.L. (2006). ATGL has a key role in lipid droplet/adiposome degradation in mammalian cells. EMBO Rep 7, 106-113.

Stone, S.J., Levin, M.C., Zhou, P., Han, J., Walther, T.C., and Farese, R.V. (2009). The endoplasmic reticulum enzyme DGAT2 is found in mitochondria-associated membranes and has a mitochondrial targeting signal that promotes its association with mitochondria. J Biol Chem 284, $5352-5361$.

Strable, M.S., and Ntambi, J.M. (2010). Genetic control of de novo lipogenesis: role in diet-induced obesity. Crit Rev Biochem Mol Biol 45, 199-214.

Su, W., Wang, Y., Jia, X., Wu, W., Li, L., Tian, X., Li, S., Wang, C., Xu, H., Cao, J., Han, Q., Xu, S., Chen, Y., Zhong, Y., Zhang, X., Liu, P., Gustafsson, J.Å., and Guan, Y. (2014). Comparative proteomic study reveals $17 \beta-$ HSD13 as a pathogenic protein in nonalcoholic fatty liver disease. Proc Natl Acad Sci USA 111, 11437-11442.

Sun, Z., Gong, J., Wu, H., Xu, W., Wu, L., Xu, D., Gao, J., Wu, J.W., Yang, H., Yang, M., and Li, P. (2013). Perilipin1 promotes unilocular lipid droplet formation through the activation of Fsp27 in adipocytes. Nat Commun 4, 1594.

Suzuki, M., Shinohara, Y., Ohsaki, Y., and Fujimoto, T. (2011). Lipid droplets: size matters. Microscopy 60, S101-S116.

Sztalryd, C., and Kimmel, A.R. (2014). Perilipins: lipid droplet coat proteins adapted for tissue-specific energy storage and utilization, and lipid cytoprotection. Biochimie 96, 96-101.

Szymanski, K.M., Binns, D., Bartz, R., Grishin, N.V., Li, W.P., Agarwal, A.K., Garg, A., Anderson, R.G.W., and Goodman, J.M. (2007). The lipodystrophy protein seipin is found at endoplasmic reticulum lipid droplet junctions and is important for droplet morphology. Proc Natl Acad Sci USA 104, 20890-20895.

Tansey, J.T., Sztalryd, C., Gruia-Gray, J., Roush, D.L., Zee, J.V., Gavrilova, O., Reitman, M.L., Deng, C.X., Li, C., Kimmel, A.R., and Londos, C. (2001). Perilipin ablation results in a lean mouse with aberrant adipocyte lipolysis, enhanced leptin production, and resistance to diet-induced obesity. Proc Natl Acad Sci USA 98, 6494-6499.

Tauchi-Sato, K., Ozeki, S., Houjou, T., Taguchi, R., and Fujimoto, T. (2002). The surface of lipid droplets is a phospholipid monolayer with a unique fatty acid composition. J Biol Chem 277, 44507-44512.

Tian, Y., Bi, J., Shui, G., Liu, Z., Xiang, Y., Liu, Y., Wenk, M.R., Yang, H., and Huang, X. (2011). Tissue-autonomous function of drosophila seipin in preventing ectopic lipid droplet formation. PLoS Genet 7, e1001364.

Toh, S.Y., Gong, J., Du, G., Li, J.Z., Yang, S., Ye, J., Yao, H., Zhang, Y., Xue, B., Li, Q., Yang, H., Wen, Z., and Li, P. (2008). Up-regulation of mitochondrial activity and acquirement of brown adipose tissue-like property in the white adipose tissue of Fsp27 deficient mice. PLoS ONE 3, e2890.

Valdearcos, M., Esquinas, E., Meana, C., Gil-de-Gomez, L., Guijas, C., Balsinde, J., and Balboa, M.A. (2011). Subcellular localization and role of lipin-1 in human macrophages. J Immunol 186, 6004-6013.

Walther, T.C., and Farese Jr, R.V. (2012). Lipid droplets and cellular lipid metabolism. Annu Rev Biochem 81, 687-714.

Wan, H.C., Melo, R.C.N., Jin, Z., Dvorak, A.M., and Weller, P.F. (2007). Roles and origins of leukocyte lipid bodies: proteomic and ultrastructural studies. FASEB J 21, 167-178.

Wang, C.W. (2016). Lipid droplets, lipophagy, and beyond. Biochim Biophys Acta $1861,793-805$.

Wang, H., Becuwe, M., Housden, B.E., Chitraju, C., Porras, A.J., Graham, M.M., Liu, X.N., Thiam, A.R., Savage, D.B., Agarwal, A.K., Garg, A., Olarte, M.J., Lin, Q., Fröhlich, F., Hannibal-Bach, H.K., Upadhyayula, S., Perrimon, N., Kirchhausen, T., Ejsing, C.S., Walther, T.C., and Farese,
R.V. (2016). Seipin is required for converting nascent to mature lipid droplets. eLife 5, e16582.

Wang, H., Hu, L., Dalen, K., Dorward, H., Marcinkiewicz, A., Russell, D., Gong, D., Londos, C., Yamaguchi, T., Holm, C., Rizzo, M.A., Brasaemle, D., and Sztalryd, C. (2009). Activation of hormone-sensitive lipase requires two steps, protein phosphorylation and binding to the PAT-1 domain of lipid droplet coat proteins. J Biol Chem 284, 32116-32125.

Wang, H., Zhang, J., Qiu, W., Han, G.S., Carman, G.M., and Adeli, K. (2011). Lipin- $1 \gamma$ isoform is a novel lipid droplet-associated protein highly expressed in the brain. FEBS Lett 585, 1979-1984.

Wang, W., Lv, N., Zhang, S., Shui, G., Qian, H., Zhang, J., Chen, Y., Ye, J., Xie, Y., Shen, Y., Wenk, M.R., and Li, P. (2012). Cidea is an essential transcriptional coactivator regulating mammary gland secretion of milk lipids. Nat Med 18, 235-243.

Watt, M.J., Holmes, A.G., Pinnamaneni, S.K., Garnham, A.P., Steinberg, G.R., Kemp, B.E., and Febbraio, M.A. (2006). Regulation of HSL serine phosphorylation in skeletal muscle and adipose tissue. AJP-Endocrinol Metab 290, E500-E508.

Watt, M.J., and Steinberg, G.R. (2008). Regulation and function of triacylglycerol lipases in cellular metabolism. Biochem J 414, 313-325.

Welte, M.A. (2015). Expanding roles for lipid droplets. Curr Biol 25, R470-R481.

Wilfling, F., Haas, J.T., Walther, T.C., and Farese, R.V. (2014). Lipid droplet biogenesis. Curr Opin Cell Biol 29, 39-45.

Wilfling, F., Wang, H., Haas, J.T., Krahmer, N., Gould, T.J., Uchida, A., Cheng, J.X., Graham, M., Christiano, R., Fröhlich, F., Liu, X., Buhman, K.K., Coleman, R.A., Bewersdorf, J., Farese Jr., R.V., and Walther, T.C. (2013). Triacylglycerol synthesis enzymes mediate lipid droplet growth by relocalizing from the ER to lipid droplets. Dev Cell 24, 384-399.

Wolinski, H., Hofbauer, H.F., Hellauer, K., Cristobal-Sarramian, A., Kolb, D., Radulovic, M., Knittelfelder, O.L., Rechberger, G.N., and Kohlwein, S.D. (2015). Seipin is involved in the regulation of phosphatidic acid metabolism at a subdomain of the nuclear envelope in yeast. Biochim Biophys Acta 1851, 1450-1464.

Wu, L., Xu, D., Zhou, L., Xie, B., Yu, L., Yang, H., Huang, L., Ye, J., Deng, H., Yuan, Y.A., Chen, S., and Li, P. (2014a). Rab8a-AS160-MSS4 regulatory circuit controls lipid droplet fusion and growth. Dev Cell 30, 378-393.

Wu, L.Z., Zhou, L.K., Chen, C., Gong, J.Y., Xu, L., Ye, J., Li, D., and Li, P. (2014b). Cidea controls lipid droplet fusion and lipid storage in brown and white adipose tissue. Sci China Life Sci 57, 107-116.

Xu, L., Zhou, L., and Li, P. (2012). CIDE proteins and lipid metabolism. Arterioscler Thromb Vasc Biol 32, 1094-1098.

Xu, W., Wu, L., Yu, M., Chen, F.J., Arshad, M., Xia, X., Ren, H., Yu, J., Xu, L., Xu, D., Li, J.Z., Li, P., and Zhou, L. (2016). Differential roles of cell death-inducing DNA fragmentation factor- $\alpha$-like effector (CIDE) proteins in promoting lipid droplet fusion and growth in subpopulations of hepatocytes. J Biol Chem 291, 4282-4293.

Yamaguchi, T., Omatsu, N., Matsushita, S., and Osumi, T. (2004). CGI-58 interacts with perilipin and is localized to lipid droplets: possible involvement of CGI-58 mislocalization in chanarin-dorfman syndrome. J Biol Chem 279, 30490-30497.

Yang, X., Lu, X., Lombès, M., Rha, G.B., Chi, Y.I., Guerin, T.M., Smart, E.J., and Liu, J. (2010). The G0/G1 switch gene 2 regulates adipose lipolysis through association with adipose triglyceride lipase. Cell Metab 11, 194-205.

Ye, J. (2013). Mechanisms of insulin resistance in obesity. Front Med 7, $14-24$.

Yu, J., Zhang, S., Cui, L., Wang, W., Na, H., Zhu, X., Li, L., Xu, G., Yang, F., Christian, M., and Liu, P. (2015). Lipid droplet remodeling and interaction with mitochondria in mouse brown adipose tissue during cold treatment. Biochim Biophys Acta 1853, 918-928.

Zechner, R. (2015). FAT FLUX: enzymes, regulators, and pathophysiology of intracellular lipolysis. EMBO Mol Med 7, 359-362.

Zechner, R., Kienesberger, P.C., Haemmerle, G., Zimmermann, R., and Lass, 
A. (2009). Adipose triglyceride lipase and the lipolytic catabolism of cellular fat stores. J Lipid Res 50, 3-21.

Zechner, R., Zimmermann, R., Eichmann, T.O., Kohlwein, S.D., Haemmerle, G., Lass, A., and Madeo, F. (2012). FAT SIGNALS-lipases and lipolysis in lipid metabolism and signaling. Cell Metab 15, 279-291.

Zehmer, J.K., Huang, Y., Peng, G., Pu, J., Anderson, R.G.W., and Liu, P. (2009). A role for lipid droplets in inter-membrane lipid traffic. Proteomics 9, 914-921.

Zhang, S., Shui, G., Wang, G., Wang, C., Sun, S., Zouboulis, C.C., Xiao, R., Ye, J., Li, W., and Li, P. (2014). Cidea control of lipid storage and secretion in mouse and human sebaceous glands. Mol Cell Biol 34, $1827-1838$.

Zhou, Z., Yon Toh, S., Chen, Z., Guo, K., Peng Ng, C., Ponniah, S., Lin, S.C., Hong, W., and Li, P. (2003). Cidea-deficient mice have lean phenotype and are resistant to obesity. Nat Genet 35, 49-56.

Zimmermann, R., Strauss, J.G., Haemmerle, G., Schoiswohl, G., Birner-Gruenberger, R., Riederer, M., Lass, A., Neuberger, G., Eisenhaber, F., Hermetter, A., and Zechner, R. (2004). Fat mobilization in adipose tissue is promoted by adipose triglyceride lipase. Science 306, 1383-1386. 